\title{
Bismuth Triflate-Chiral Bipyridine Complexes as Water-Compatible Chiral Lewis
}

Acids

Shū Kobayashi, ${ }^{*}$ Tsuyoshi Ogino, Haruka Shimizu, Shunpei Ishikawa, Tomoaki Hamada, and Kei Manabe

Graduate School of Pharmaceutical Sciences, The University of Tokyo, The HFRE Division ERATO, Japan Science and Technology Agency (JST), Hongo, Bunkyo-ku, Tokyo 113-0033, Japan.

\section{Supporting Information}

Table of Contents

Pages

Experimental S-1

Typical Experimental Procedures for the Hydroxymethylation

Characterization Data of the Hydroxymethylated Adducts

Crystallization of $\left[\mathrm{BiBr}_{3} \bullet \mathbf{1}\right] \bullet\left(\mathrm{H}_{2} \mathrm{O}\right)_{2} \cdot \mathrm{DME}$ Complexes

NMR Analyses of the Catalyst Structures

References

S-8

\section{Experimental}

General: Melting points were uncorrected. $\quad{ }^{1} \mathrm{H}$ and ${ }^{13} \mathrm{C}$ NMR spectra were recorded on a JEOL JNM-LA300, JNM-LA400, ECX-400 or ECX-600 spectrometer in $\mathrm{CDCl}_{3}$ unless otherwise noted. Tetramethylsilane (TMS) served as an internal standard $(\delta=0$ ppm) for ${ }^{1} \mathrm{H}$ NMR and $\mathrm{CDCl}_{3}$ as an internal standard $(\delta=77.0 \mathrm{ppm})$ for ${ }^{13} \mathrm{C} \mathrm{NMR}$. Preparative thin-layer chromatography was carried out using Wakogel B-5F. An aqueous solution of formaldehyde was purchased from Wako Pure Chemical Industries, Ltd. 
Typical Experimental Procedure for the Hydroxymethylation of Propiophenone-derived Silicon Enolate: A mixture of $\mathrm{Bi}(\mathrm{OTf})_{3}(14 \mathrm{mg}, 0.020$ $\mathrm{mmol})$ and 1 (20 mg, $0.060 \mathrm{mmol})$ in DME (1 mL) was stirred at room temperature for $30 \mathrm{~min}$ to afford $20 \mathrm{mM}$ of a catalyst solution. A reaction vessel charged with the catalyst solution $(150 \mu \mathrm{L})$ was cooled at $0{ }^{\circ} \mathrm{C}$ for $10 \mathrm{~min}$, and an $81 \mathrm{mM}$ solution of 2,2'-bipyridine in DME $(185 \mu \mathrm{L})$ was added. Then an aqueous solution of formaldehyde $(129 \mathrm{mg}, 35 \% \mathrm{w} / \mathrm{w}, 1.5 \mathrm{mmol})$ and the silicon enolate $(62 \mathrm{mg}, 0.30$ mmol) were added to the mixture. The whole was stirred until the silicon enolate disappeared completely (checked by TLC). The reaction was quenched with saturated aqueous $\mathrm{NaHCO}_{3}$. The resultant mixture was extracted with dichloromethane (3 times), and the combined organic layers were dried over anhydrous $\mathrm{Na}_{2} \mathrm{SO}_{4}$. The solvents were evaporated, and the residue was purified by preparative TLC (silica gel, hexane/ethyl acetate (2/1)) to give the hydroxymethylated adduct. The enantiomeric excess of the product was determined by chiral HPLC analysis.

\section{Characterization Data of the Hydroxymethylated Adducts}

(S)-3-Hydroxy-2-methyl-1-phenylpropan-1-one ${ }^{1}$<smiles>CC(CO)C(=O)c1ccccc1</smiles>

${ }^{1} \mathrm{H}$ NMR $\left(\mathrm{CDCl}_{3}\right) \delta 1.24$ (d, 3H, $J=7.1 \mathrm{~Hz}$ ), 2.35 (brs), 3.68 (ddq, $1 \mathrm{H}$, $J=4.3,7.0,7.1 \mathrm{~Hz}), 3.80(\mathrm{dd}, 1 \mathrm{H}, J=4.3,11.1 \mathrm{~Hz}), 3.94(\mathrm{dd}, 1 \mathrm{H}, J=$ 7.0, 11.1 Hz), $7.48(\mathrm{dd}, 2 \mathrm{H}, J=7.3,8.5 \mathrm{~Hz}), 7.58(\mathrm{t}, 1 \mathrm{H}, J=7.3 \mathrm{~Hz})$, $7.97(\mathrm{~d}, 2 \mathrm{H}, J=8.5 \mathrm{~Hz}) ;{ }^{13} \mathrm{C} \mathrm{NMR}\left(\mathrm{CDCl}_{3}\right) \delta 14.5,42.9,64.5,128.4,128.7,133.3$, 136.1, 204.4; HPLC (Daicel Chiralpak AD-H, hexane $/ i$-PrOH $=19 / 1$, flow rate $=1.0$ $\mathrm{mL} / \mathrm{min}) t_{\mathrm{R}}=17.5 \min ($ major, $S), t_{\mathrm{R}}=20.3 \min ($ minor, $R$ ).

\section{2-(Hydroxymethyl)-1-phenylbutan-1-one ${ }^{3}$}<smiles>CCC(CO)C(=O)c1ccccc1</smiles>

${ }^{1} \mathrm{H}$ NMR $\left(\mathrm{CDCl}_{3}\right) \delta 0.95(\mathrm{t}, 3 \mathrm{H}, J=7.4 \mathrm{~Hz}), 1.61-1.83(\mathrm{~m}, 2 \mathrm{H})$, 2.40 (brs), 3.52-3.58 (m, 1H), $3.84(\mathrm{dd}, 1 \mathrm{H}, J=3.9,11.0 \mathrm{~Hz}), 3.98$ $(\mathrm{dd}, 1 \mathrm{H}, J=6.8,11.0 \mathrm{~Hz}), 7.45-7.50(\mathrm{~m}, 2 \mathrm{H}), 7.56-7.60(\mathrm{~m}, 1 \mathrm{H})$, 7.95-7.97 (m, 2H); ${ }^{13} \mathrm{C} \mathrm{NMR}\left(\mathrm{CDCl}_{3}\right) \delta 11.8,22.3,49.5,62.6,128.3,128.7,133.2$, 136.8, 204.6; IR (neat) 3435, 2966, 1678, 1043, $704 \mathrm{~cm}^{-1}$; HRMS (ESI-TOF) calcd for $\mathrm{C}_{11} \mathrm{H}_{14} \mathrm{O}_{2} \mathrm{Na}\left([\mathrm{M}+\mathrm{Na}]^{+}\right): 201.0891$, found: 201.0889; $[\alpha]^{24}{ }_{\mathrm{D}}-0.6\left(c 0.75, \mathrm{CHCl}_{3}\right)(91 \%$ ee); HPLC (Daicel Chiralpak AD-H, hexane $/ i-\mathrm{PrOH}=19 / 1$, flow rate $=1.0 \mathrm{~mL} / \mathrm{min}$ ) $t_{\mathrm{R}}$ $=18.8 \min$ (major), $t_{\mathrm{R}}=22.4 \min$ (minor). 


\section{3-(Hydroxy)-2-methyl-1-(4-methoxyphenyl)propan-1-one}

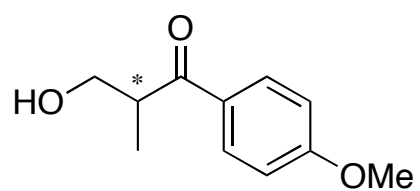

${ }^{1} \mathrm{H}$ NMR $\left(\mathrm{CDCl}_{3}\right) \delta 1.22(\mathrm{~d}, 3 \mathrm{H}, J=7.1 \mathrm{~Hz}), 2.60$ (brs), 3.62 (ddq, $1 \mathrm{H}, J=4.3,7.0,7.1 \mathrm{~Hz}), 3.78(\mathrm{dd}, 1 \mathrm{H}, J=4.3,11 \mathrm{~Hz})$,

$3.87(\mathrm{~s}, 3 \mathrm{H}), 3.91(\mathrm{dd}, 1 \mathrm{H}, J=7.0,11 \mathrm{~Hz}), 6.95(\mathrm{~d}, 2 \mathrm{H}, J=$ $8.9 \mathrm{~Hz}), 7.95(\mathrm{~d}, 2 \mathrm{H}, J=8.9 \mathrm{~Hz}) ;{ }^{13} \mathrm{C} \mathrm{NMR}\left(\mathrm{CDCl}_{3}\right) \delta 14.7,42.4,55.4,64.6,113.8$, 129.0, 130.7, 163.6, 202.9; IR (neat) 3438, 2933, 1667, 1597, 1251, 1173, 1047, 975, $843 \mathrm{~cm}^{-1}$; MS m/z $194\left(\mathrm{M}^{+}\right)$; Anal. Calcd for $\mathrm{C}_{11} \mathrm{H}_{14} \mathrm{O}_{3}: \mathrm{C}, 68.02 ; \mathrm{H}, 7.27$. found: $\mathrm{C}$, 67.88; H, 7.33; $[\alpha]^{24}{ }_{\mathrm{D}}+4.2\left(c\right.$ 1.5, $\left.\mathrm{CHCl}_{3}\right)(88 \%$ ee); HPLC (Daicel Chiralpak AD-H, hexane $/ i-\mathrm{PrOH}=19 / 1$, flow rate $=1.0 \mathrm{~mL} / \mathrm{min}$ ) $t_{\mathrm{R}}=30.7 \mathrm{~min}$ (major), $t_{\mathrm{R}}=38.9 \mathrm{~min}$ (minor).

\section{3-(Hydroxy)-2-methyl-1-(4-chlorophenyl)propan-1-one}<smiles>CC(CO)C(=O)c1ccc(Cl)cc1</smiles>

${ }^{1} \mathrm{H}$ NMR $\left(\mathrm{CDCl}_{3}\right) \delta 1.21(\mathrm{~d}, 3 \mathrm{H}, J=7.3 \mathrm{~Hz}), 2.52$ (brs), 3.62 (ddq, $1 \mathrm{H}, J=4.3,7.2,7.3 \mathrm{~Hz}), 3.77(\mathrm{dd}, 1 \mathrm{H}, J=4.3,11 \mathrm{~Hz}$ ), $3.87(\mathrm{~s}, 3 \mathrm{H}), 3.93(\mathrm{dd}, 1 \mathrm{H}, J=7.2,11 \mathrm{~Hz}), 7.44(\mathrm{~d}, 2 \mathrm{H}, J=8.5$ $\mathrm{Hz}), 7.90(\mathrm{~d}, 2 \mathrm{H}, J=8.5 \mathrm{~Hz}) ;{ }^{13} \mathrm{C} \mathrm{NMR}\left(\mathrm{CDCl}_{3}\right) \delta 14.4,43.0,64.4,129.0,129.8,134.4$, 139.7, 203.0; IR (neat) 3424, 2937, 1682, 1590, 1401, 1092, 976, $841 \mathrm{~cm}^{-1}$; MS m/z 198 $\left(\mathrm{M}^{+}\right)$; Anal. Calcd for $\mathrm{C}_{10} \mathrm{H}_{11} \mathrm{ClO}_{2}$ : C, 60.48; H, 5.58. found: $\mathrm{C}, 60.18 ; \mathrm{H}, 5.85 ;[\alpha]^{24} \mathrm{D}$ +4.1 (c 1.05, $\left.\mathrm{CHCl}_{3}\right)$ (89\% ee); HPLC (Daicel Chiralpak AD-H, hexane/i-PrOH = 19/1, flow rate $=1.0 \mathrm{~mL} / \mathrm{min}$ ) $t_{\mathrm{R}}=18.6 \mathrm{~min}$ (major), $t_{\mathrm{R}}=28.7 \mathrm{~min}$ (minor).

\section{3-Hydroxy-1-(2-methoxynaphthalen-6-yl)-2-methylpropan-1-one}

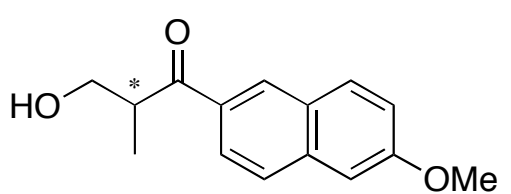

${ }^{1} \mathrm{H} \mathrm{NMR}\left(\mathrm{CDCl}_{3}\right) \delta 1.28(\mathrm{~d}, 3 \mathrm{H}, J=6.9 \mathrm{~Hz}), 2.60(\mathrm{~s}$, $1 \mathrm{H}), 3.78-3.86(\mathrm{~m}, 2 \mathrm{H}), 3.93(\mathrm{~s}, 3 \mathrm{H}), 3.98(\mathrm{~m}, 1 \mathrm{H}), 7.13$ $(\mathrm{m}, 1 \mathrm{H}), 7.20(\mathrm{~m}, 1 \mathrm{H}), 7.75(\mathrm{~m}, 1 \mathrm{H}), 7.83(\mathrm{~m}, 1 \mathrm{H}), 7.98$ $(\mathrm{m}, 1 \mathrm{H}), 8.40(\mathrm{~m}, 1 \mathrm{H}) ;{ }^{13} \mathrm{C} \mathrm{NMR}\left(\mathrm{CDCl}_{3}\right) \delta 14.8,42.7,55.4,64.7,105.7,119.7,124.7$, 127.2, 127.8, 130.1, 131.2, 131.4, 137.4, 159.8, 204.1; IR (neat) 3444, 1665, 1622, 1477 , 1268, 1187, $1028 \mathrm{~cm}^{-1}$; HRMS (ESI-TOF) calcd for $\mathrm{C}_{15} \mathrm{H}_{17} \mathrm{O}_{3}\left([\mathrm{M}+\mathrm{H}]^{+}\right): 245.1178$, found: 245.1166; $\quad[\alpha]^{24} \mathrm{D}+2.3\left(c\right.$ 1.84, $\left.\mathrm{CHCl}_{3}\right)(92 \%$ ee); HPLC (Daicel Chiralcel OD, hexane $/ i-\mathrm{PrOH}=19 / 1$, flow rate $=1.0 \mathrm{~mL} / \mathrm{min}) t_{\mathrm{R}}=30.7 \mathrm{~min}($ minor $), t_{\mathrm{R}}=36.6 \mathrm{~min}$ (major). 


\section{2,3-Dihydro-2-(hydroxymethyl)-2-methylinden-1-one ${ }^{3}$}

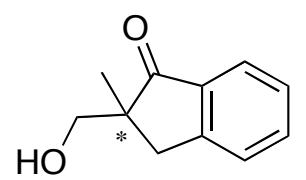

${ }^{1} \mathrm{H} \mathrm{NMR}\left(\mathrm{CDCl}_{3}\right) \delta 1.26(\mathrm{~s}, 3 \mathrm{H}), 2.91(\mathrm{~d}, 1 \mathrm{H}, J=7.2 \mathrm{~Hz}), 3.24(\mathrm{~d}$, $1 \mathrm{H}, J=7.2 \mathrm{~Hz}), 3.64(\mathrm{~d}, 1 \mathrm{H}, J=11 \mathrm{~Hz}), 3.83(\mathrm{~d}, 1 \mathrm{H}, J=11 \mathrm{~Hz})$, 7.35-7.41 (m, 1H), 7.45-7.48 (m, 1H), 7.59-7.64 (m, 1H), 7.64-7.77 $(\mathrm{m}, 1 \mathrm{H}) ;{ }^{13} \mathrm{C} \mathrm{NMR}\left(\mathrm{CDCl}_{3}\right) \delta 20.7,38.0,50.8,67.8,124.2,126.7,127.5,135.2,135.8$, 153.2, 211.1; IR (KBr) 3473, 2962, 2921, 2866, $1703 \mathrm{~cm}^{-1}$; Mp 83-89 ${ }^{\circ} \mathrm{C}$; Anal. calcd for $\mathrm{C}_{11} \mathrm{H}_{12} \mathrm{O}_{2}: \mathrm{C}, 74.98 ; \mathrm{H}, 6.86$. found: $\mathrm{C}, 74.80 ; \mathrm{H}, 7.05 ; \quad[\alpha]^{24}{ }_{\mathrm{D}}+1.6\left(c 0.95, \mathrm{CHCl}_{3}\right)$ (88\% ee); HPLC (Daicel Chiralcel OB-H, hexane $/ i-\mathrm{PrOH}=100 / 1$, flow rate $=1.0$ $\mathrm{mL} / \mathrm{min}$ ) $t_{\mathrm{R}}=39.6 \min$ (major), $t_{\mathrm{R}}=50.0 \min$ (minor).

\section{3,4-Dihydro-2-(hydroxymethyl)-2-methylnaphthalen-1(2H)-one ${ }^{3}$}<smiles>CC1(CO)CCc2ccccc2C1=O</smiles>

${ }^{1} \mathrm{H}$ NMR $\left(\mathrm{CDCl}_{3}\right) \delta 1.23$ (s, 3H), $1.78(\mathrm{ddd}, 1 \mathrm{H}, J=3.7,4.9,13.4$ Hz), 2.24 (ddd, 1H, $J=5.1,12.0,13.4 \mathrm{~Hz}$ ), 2.77 (brs), 2.94 (ddd, $1 \mathrm{H}, J=3.7,5.1,17.3 \mathrm{~Hz}), 3.15(\mathrm{ddd}, 1 \mathrm{H}, J=4.9,12.0,17.3 \mathrm{~Hz})$, $3.65(\mathrm{~d}, 1 \mathrm{H}, J=11.2 \mathrm{~Hz}), 3.74(\mathrm{~d}, 1 \mathrm{H}, J=11.2 \mathrm{~Hz}), 7.24-7.26(\mathrm{~m}, 1 \mathrm{H}), 7.29-7.33(\mathrm{~m}$, $1 \mathrm{H}), 7.47-7.50(\mathrm{~m}, 1 \mathrm{H}), 8.00-8.03(\mathrm{~m}, 1 \mathrm{H}) ;{ }^{13} \mathrm{C} \mathrm{NMR}\left(\mathrm{CDCl}_{3}\right) \delta$ 18.2, 25.0, 31.3, 46.3, 69.0, 126.7, 127.7, 128.7, 131.4, 133.6, 143.4, 204.0; IR (neat) 3456, 2931, 1678, 1047, $742 \mathrm{~cm}^{-1}$; HRMS (ESI-TOF) calcd for $\mathrm{C}_{12} \mathrm{H}_{14} \mathrm{O}_{2} \mathrm{Na}\left([\mathrm{M}+\mathrm{Na}]^{+}\right): 213.0891$, found: 213.0881; $[\alpha]^{24}{ }_{\mathrm{D}}-1.4$ (c 1.3, $\mathrm{CHCl}_{3}$ ) (95\% ee); HPLC (Daicel Chiralcel OD, hexane $/ i-\mathrm{PrOH}=100 / 1$, flow rate $=1.0 \mathrm{~mL} / \mathrm{min}$ ) $t_{\mathrm{R}}=28.6 \mathrm{~min}$ (major), $t_{\mathrm{R}}=34.3 \mathrm{~min}$ (minor).

\section{2-Butyl-3,4-dihydro-2-(hydroxymethyl)naphthalen-1(2H)-one}

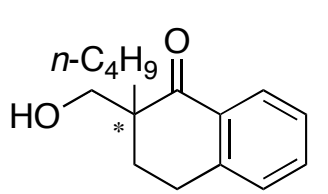

${ }^{1} \mathrm{H}$ NMR $\left(\mathrm{CDCl}_{3}\right) \delta ;{ }^{13} \mathrm{C} \mathrm{NMR}\left(\mathrm{CDCl}_{3}\right) \delta 13.9,23.2,24.9,25.8$, $28.0,29.9,49.5,66.5,126.7,127.7,128.7,131.6,133.5,143.4$, 204.4; IR (neat) 3443, 1672, 1458, 1227, 1053, $742 \mathrm{~cm}^{-1}$; HRMS (ESI-TOF) calcd for $\mathrm{C}_{15} \mathrm{H}_{21} \mathrm{O}_{2} \mathrm{Na}\left([\mathrm{M}+\mathrm{Na}]^{+}\right): 255.1356$, found: $255.1362 ;[\alpha]^{24} \mathrm{D}-2.8(c$ $0.75, \mathrm{CHCl}_{3}$ ) (93\% ee); HPLC (Daicel Chiralcel OD, hexane/i-PrOH $=100 / 1$, flow rate $=1.0 \mathrm{~mL} / \mathrm{min}$ ) $t_{\mathrm{R}}=20.6 \min$ (major), $t_{\mathrm{R}}=23.6 \min$ (minor). 
(R)-2-(Hydroxymethyl)-2-methylcyclohexanone ${ }^{2}$

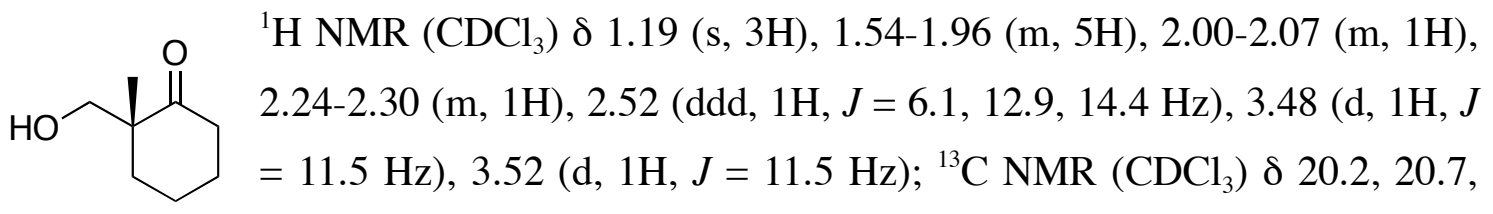
27.3, 35.5, 38.9, 50.1, 69.0, 218.1 .

\section{[(R)-1-Methyl-2-oxocyclohexyl]methyl benzoate ${ }^{3}$}

To a solution of 2-(hydroxymethyl)-2-methylcyclohexanone (14 $\mathrm{mg}$,
$0.099 \mathrm{mmol})$ in $\mathrm{CH}_{2} \mathrm{Cl}_{2}(0.99 \mathrm{~mL})$ were added benzoyl chloride $(23 \mu \mathrm{L}$,
$0.20 \mathrm{mmol})$ and pyridine $(40 \mu \mathrm{L}, 0.49 \mathrm{mmol})$ at room temperature. After stirring for $2 \mathrm{~h}$, the reaction was quenched with water. The resultant mixture was extracted with $\mathrm{CH}_{2} \mathrm{Cl}_{2}$ (twice), and the combined organic layers were washed with brine and dried over anhydrous $\mathrm{Na}_{2} \mathrm{SO}_{4}$. The solvents were evaporated, and the residue was purified by preparative TLC (silica gel, hexane/ethyl acetate (6/1)) to give the desired product ( $75 \%$ yield).

${ }^{1} \mathrm{H} \mathrm{NMR}\left(\mathrm{CDCl}_{3}\right) \delta 1.26(\mathrm{~s}, 3 \mathrm{H}), 1.73-1.98(\mathrm{~m}, 6 \mathrm{H}), 2.42-2.54(\mathrm{~m}, 2 \mathrm{H}), 4.38(\mathrm{~d}, 1 \mathrm{H}, J=$ $11.0 \mathrm{~Hz}), 4.46(\mathrm{~d}, 1 \mathrm{H}, J=11.0 \mathrm{~Hz}), 7.41-7.45(\mathrm{~m}, 2 \mathrm{H}), 7.53-7.58(\mathrm{~m}, 1 \mathrm{H}), 7.99-8.01(\mathrm{~m}$, $2 \mathrm{H}) ;{ }^{13} \mathrm{C} \mathrm{NMR}\left(\mathrm{CDCl}_{3}\right) \delta 20.8,21.0,27.2,36.4,38.8,48.9,69.1,128.3,129.5,130.0$, 133.0, 166.3, 212.9; IR (neat) 2937, 1718, 1274, 1115, $714 \mathrm{~cm}^{-1}$; Anal. calcd for $\mathrm{C}_{15} \mathrm{H}_{18} \mathrm{O}_{3}$ : C, 73.15; H, 7.37. found: C, 73.24; $\mathrm{H}, 7.54 ;[\alpha]^{18} \mathrm{D}-0.2\left(c 0.50, \mathrm{CHCl}_{3}\right.$ ) (for $R, 77 \%$ ee); HPLC (Daicel Chiralpak AD-H, hexane $/ i-\mathrm{PrOH}=100 / 1$, flow rate $=0.5$ $\mathrm{mL} / \min ) t_{\mathrm{R}}=39.7 \min ($ major, $R), t_{\mathrm{R}}=42.3 \min ($ minor,$S)$.

\section{2-Benzyl-2-(hydroxymethyl)cyclohexanone ${ }^{3}$}

${ }^{1} \mathrm{H} N M R\left(\mathrm{CDCl}_{3}\right) \delta 1.40-1.48(\mathrm{~m}, 1 \mathrm{H}), 1.60-1.78(\mathrm{~m}, 3 \mathrm{H}), 1.91-2.11(\mathrm{~m}$,
$2 \mathrm{H}), 2.35-2.41(\mathrm{~m}, 1 \mathrm{H}), 2.55-2.80(\mathrm{~m}, 2 \mathrm{H}), 2.88(\mathrm{~d}, 1 \mathrm{H}, J=13.4 \mathrm{~Hz})$,
$3.08(\mathrm{~d}, 1 \mathrm{H}, J=13.4 \mathrm{~Hz}), 3.34(\mathrm{~d}, 1 \mathrm{H}, J=11.6 \mathrm{~Hz}), 3.46(\mathrm{~d}, 1 \mathrm{H}, J=$ 11.6 Hz), 7.21-7.31 (m, 5H); ${ }^{13} \mathrm{C} \mathrm{NMR}\left(\mathrm{CDCl}_{3}\right) \delta$ 20.6, 27.0, 31.5, 37.0, 39.3, 53.8, 65.6, 126.6, 128.2, 130.5, 135.9, 217.9; IR (neat) 3452, 2941, 1699, 1454, 1061, $706 \mathrm{~cm}^{-1}$; HRMS (ESI-TOF) calcd for $\mathrm{C}_{14} \mathrm{H}_{18} \mathrm{O}_{2} \mathrm{Na}\left([\mathrm{M}+\mathrm{Na}]^{+}\right)$: 241.1204, found: 241.1204; $[\alpha]^{24}{ }_{\mathrm{D}}-8.2\left(c\right.$ 1.25, $\left.\mathrm{CHCl}_{3}\right)(82 \%$ ee); HPLC (Daicel Chiralpak AD-H, hexane/i-PrOH $=40 / 1$, flow rate $=1.0 \mathrm{~mL} / \mathrm{min}$ ) $t_{\mathrm{R}}=22.1 \mathrm{~min}$ (major), $t_{\mathrm{R}}=29.8 \mathrm{~min}$ (minor). 


\section{2-Benzyl-2-(hydroxymethyl)cyclopentanone}

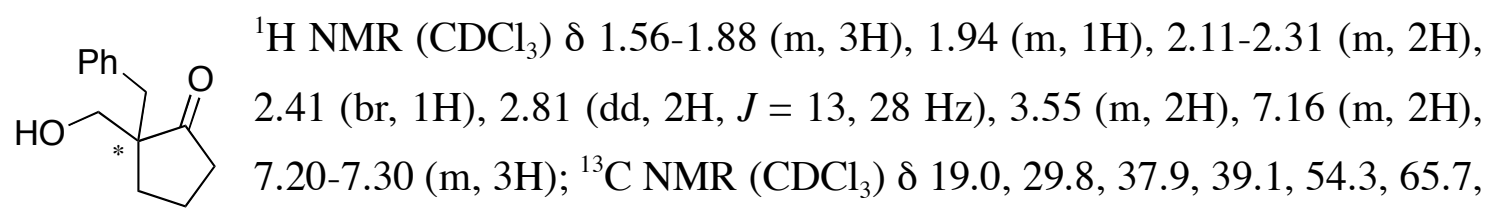

126.6, 128.3, 130.2, 136.8, 224.5; IR (neat) 3438, 1727, 1160, 1053, 757, $705 \mathrm{~cm}^{-1}$; HRMS (ESI-TOF) calcd for $\mathrm{C}_{10} \mathrm{H}_{16} \mathrm{O}_{2} \mathrm{Na}\left([\mathrm{M}+\mathrm{Na}]^{+}\right)$: 227.1043, found: 227.1040; $[\alpha]^{24} \mathrm{D}-4.5\left(c\right.$ 0.85, $\left.\mathrm{CHCl}_{3}\right)(79 \%$ ee); HPLC (Daicel Chiralpak AD-H, hexane/i-PrOH $=19 / 1$, flow rate $=1.0 \mathrm{~mL} / \mathrm{min}$ ) $t_{\mathrm{R}}=15.1 \mathrm{~min}$ (major), $t_{\mathrm{R}}=19.0 \mathrm{~min}$ (minor) .

\section{Crystallization of $\left[\mathrm{BiBr}_{3} \bullet 1\right] \bullet\left(\mathrm{H}_{2} \mathrm{O}\right)_{2} \cdot \mathrm{DME}$ complexes}

Crystallization of $\left[\mathrm{BiBr}_{3} \bullet \mathbf{1}\right] \cdot\left(\mathbf{H}_{2} \mathbf{O}\right)_{2} \bullet \mathbf{D M E}$ was carried out as follows: To a yellow solution of $\mathrm{BiBr}_{3}(20.2 \mathrm{mg}, 45 \mu \mathrm{mol})$ in DME (20 $\left.\mu \mathrm{L}\right)$ was added 1 (14.8 mg, $\left.45 \mu \mathrm{mol}\right)$ at room temperature. The solution was warmed to $75{ }^{\circ} \mathrm{C}$ (oil bath temperature). Then, a white precipitate was gradually formed, and was dissolved by addition of DME $(630 \mu \mathrm{L})$. The resulting solution was allowed to stand at room temperature under DME vapor to give the crystals.

\section{NMR analysis of the catalyst structure}

NMR experiments were conducted to obtain some information about the catalyst structure when the $\mathrm{Bi}(\mathrm{OTf})_{3}-1$ ratio were 1:0.5, 1:1, 1:2 and 1:3 (Figure S-1). When the $\mathrm{Bi}(\mathrm{OTf})_{3}$ and 1 were combined at the ratio of $1: 0.5$, a signal at $5.49 \mathrm{ppm}$ was dominant. On the other hand, another signal at $4.72 \mathrm{ppm}$ increased when the ratio of 1 to $\mathrm{Bi}(\mathrm{OTf})_{3}$ increased, and finally the former signal disappeared when $\mathrm{Bi}(\mathrm{OTf})_{3}$ was combined with three equivalents of $\mathbf{1}$. These results indicate that two equivalents of $\mathrm{Bi}(\mathrm{OTf})_{3}$ and one equivalent of $\mathbf{1}$ formed complex $\mathbf{3}$, and that complex $\mathbf{4}$ consisting of one equivalent of $\mathrm{Bi}(\mathrm{OTf})_{3}$ and one equivalent of $\mathbf{1}$ was generated when excess amount of 1 was added (Scheme S-1). 
Figure S-1. ${ }^{1} \mathrm{H}$ NMR analysis of the catalyst structure.

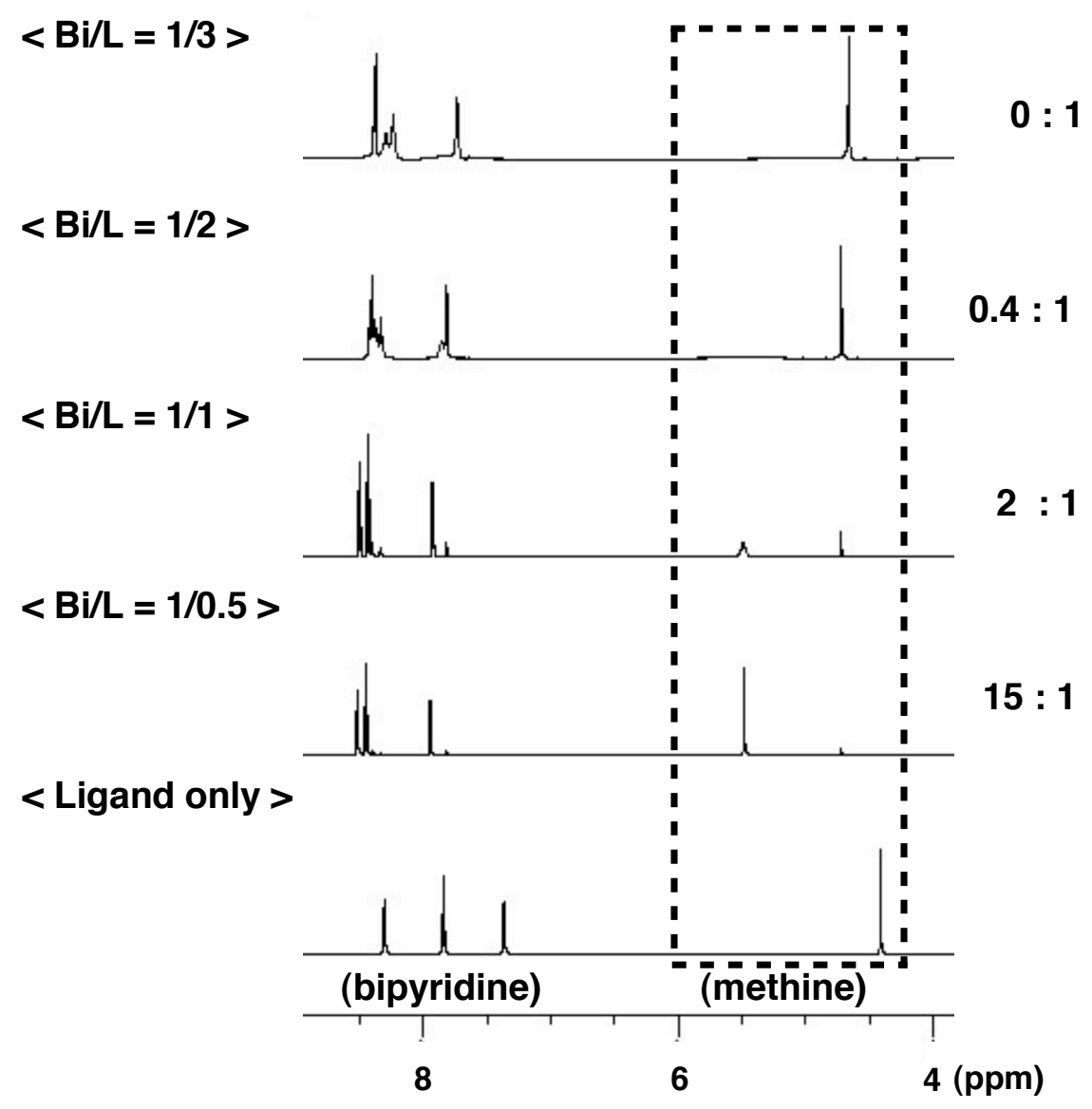

Scheme S-1.

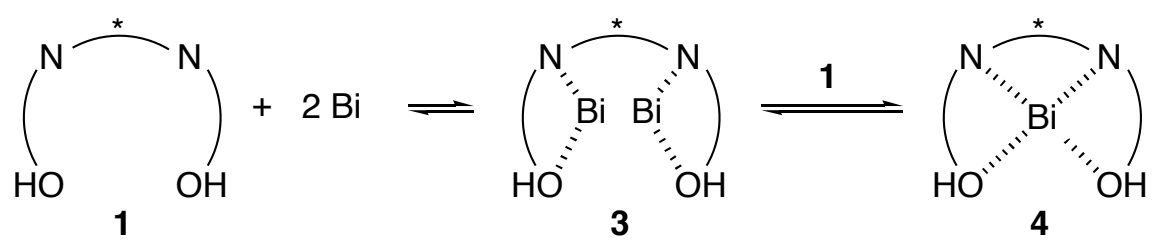

Representative Procedure for NMR Experiments: $\quad\left[\mathrm{Bi}(\mathrm{OTf})_{3}: \mathbf{1}=1: 1\right] \quad$ A solution of $\mathrm{Bi}(\mathrm{OTf})_{3}(24 \mathrm{mg}, 0.036 \mathrm{mmol})$ and $1(12 \mathrm{mg} 0.036 \mathrm{mmol})$ in $\mathrm{CD}_{3} \mathrm{CN}(900 \mu \mathrm{L})$ was stirred at $0{ }^{\circ} \mathrm{C}$ for $10 \mathrm{~min}$. $\quad \mathrm{D}_{2} \mathrm{O}(100 \mu \mathrm{L})$ was added to the mixture, and the solution was stirred for $30 \mathrm{~min}$ at $0{ }^{\circ} \mathrm{C}$. NMR experiments were conducted at $0{ }^{\circ} \mathrm{C}$ using ECX-600 NMR spectrometer. The deuterated solvents were purchased from Cambridge Isotope Laboratories, Inc. and used without further purification. 


\section{References}

(1) Manabe, K.; Ishikawa, S.; Hamada, T.; Kobayashi, S. Tetrahedron 2003, 59, 10439.

(2) Miyaoka, H.; Kajiwara, Y.; Hara, M.; Suma, A.; Yamada, Y. Tetrahedron: Asymmetry 1999, $10,3189$.

(3) Ishikawa, S.; Hamada, T.; Manabe, K.; Kobayashi, S. J. Am. Chem. Soc. 2004, 126, 12236. 